MS15-03

\section{Crystallographic characterization and 3D modeling of synthetic plagioclases: a better understanding of morphologies, connectivity and size distribution depending on temperature.}

Claire Charles ${ }^{1}$, Elsa Ottavi-Pupier ${ }^{1}$, Julien Duquennoy ${ }^{1}$, Michihiko Nakamura $^{2}$, Satoshi Okumura ${ }^{2}$, Akira Tsuchiyama ${ }^{3}$, Kentaro Uesugi ${ }^{4}$, Masayuki Uesugi ${ }^{5}$

1. Geosciences Department Institut Polytechnique UniLaSalle, Beauvais, France

2. Department of Earth Science, Tohoku University, Tohoku, Japan

3. Department of Geology and Mineralogy, Kyoto University, Kyoto, Japan

4. Spring-8/JASRI, Koto, Japan

5. JSPEC/JAXA, Tsukuba, Japan

email: claire.charles21@gmail.com

Experimental samples of basaltic composition, at different temperatures, were created in laboratory. Synthetic basalt was obtained by cooling control (rate $1{ }^{\circ} \mathrm{C} / \mathrm{h}$ ) until $40 \%$ of crystallization [1]. In-situ observations of plagioclase crystals were made using X-ray micro-tomography beam lines at SPring-8. Grey levels between glass and plagioclases are close and lots of plagioclases are connected, complicating the automatic image treatment. Moreover using usual software for CT treatment, the images are processed automatically but this method generates problems like the data suppression and the calculation approximations (volume, connectivity, size...).

During this study, three dimensions plagioclase crystals resulting from the experimental samples were designed. The $3 \mathrm{D}$ reconstruction has been conducted using the numerical platform 3DEXPERIENCEC developed by Dassault Systèmes (DS). The modeling is carried out manually by working on successive images of the samples in order to observe all the crystals and to consider how they are spatially organized. Thus, sections of crystals have been drawn and linked together to build the crystal shape in 3D.

As the result, all the experimental samples were modeled. The 3D reconstructions (figure) allowed to visualize the textures and the interactions between crystals depending on the temperatures. This has permitted to determine the connectivity and the crystallinity of the samples. Moreover, size measurements on axes and volume measurements of each modeled crystal were performed. The plagioclase 3D Crystal Size Distributions (CSD) with the major axis data and with the minor axis data were calculated. The CSD law expresses the number of crystals per interval size per unit volume [2]. This physical law is aimed at connecting size, distribution and abundance of crystals in a rock to the thermal history. CSD of this study are compared with calculated CSD from previous experimental studies using others methods: 2D modeling and 3D mathematic conversions [1] or 3D modeling with different software and automatic axis calculations [3]. The results show similar curves with: a decrease in the CSD according to the temperature and comparable slopes.

The numerical platform 3DEXPERIENCEC provides many advantages for crystallographic studies, calculation and vi- sualization. All this work on the physical parameters of the plagioclases has enabled to understand their evolution depending on the temperatures.

References:

[1] Pupier et al. (2008). Cont. Min. Pet., 155, 555-570.

[2] Marsh, B. D. (1988). Cont. Min. Pet., 99, 277-291.

[3] Duchêne et al. (2008). Am. Min., 93, 893-901.

Keywords: "3D modelin" "characterization" "crystals" 